\title{
Supramolecular dendritic polymers for diagnostic and theranostic applications
}

\author{
Yan $\mathrm{Wu}^{1}$, Guanzhe $\mathrm{Xu}^{2}$, Xin $\mathrm{Jin}^{2 *}$ and Xinyuan $\mathrm{Zhu}^{2^{*}}$
}

\begin{abstract}
Supramolecular dendritic polymers (SDPs) provide a new opportunity for the precision diagnosis and treatment of diseases. SDPs are a novel class of non-covalently bonded macromolecules with highly branched structure and three-dimensional globular topology, which exhibit dynamic/ reversible features and unique physical/chemical properties (e.g., high solubility, low viscosity, and numerous functional terminal groups). The reversibility of non-covalent interactions endows SDPs with the ability of facile preparation, smart responsiveness, and simple metabolism. These special characteristics determine the properties of SDPs, which are the key points for theranostic applications, including diagnosis, therapy, and theranostics. In this review, we briefly summarize the design and synthesis of SDPs with aimed structures, properties, functions as well as their present diagnostic and theranostic applications. These developments on the preparation and applications of SDPs for diagnostic and theranostic purpose promote interdisciplinary research fields of chemistry, material and biomedical science.
\end{abstract}

Keywords: dendritic polymers, supramolecular polymers, diagnosis, therapy, theranostics

\section{INTRODUCTION}

The diagnosis and therapy of various diseases have attracted more and more attention in recent years. To realize an efficient treatment, biocompatible and biodegradable polymers are frequently used as carriers, probes or therapeutic agents because of their macromolecular scale and smart responsiveness. Comparing to the traditional linear polymers, dendritic polymers exhibit treelike topological structures, a high density of terminal groups, and precise spatial control [1-3]. Therefore, dendritic polymers show great potential in various biomedical fields, especially for the diagnosis and therapy. However, there are still several obstacles for their diagnostic and theranostic applications: (1) multiple and complicated synthetic and purification steps of dendritic polymers result in ill-defined structure, low scalability and high cost; (2) uncontrollability of the ratio of diagnostic probes and therapeutic agents in dendritic polymers induces the poor reproducibility; (3) inconclusive bio-safety of dendritic polymers due to their potential toxicity, unpredicted bioelimination and long-term invivo effect limits their clinical transformation [4,5].

The appearance of supramolecular dendritic polymers (SDPs) provides a new opportunity for solving the abovementioned problems. As an elegant bridge to combine supramolecular chemistry and dendritic polymer, SDPs show dynamic/reversible features and unique physical/chemical properties [6]. Owing to the introduction of non-covalent interactions into polymeric backbone, SDPs are endowed with several particular advantages: facile synthesis and purification, switchable structures and shapes, and smart stimuli-responsiveness [7-9]. Different from conventional dendritic polymers, SDPs have a large number of complementary binding sites for building blocks because of their topological structures, which influences their morphology and functions. The highly branched three-dimensional architectures of SDPs result in attractive properties such as little molecular entanglement, low viscosity, high solubility, and a lot of functional terminal groups. Usually, hydrogen bonding, host-guest interaction, metal-ligand

${ }^{1}$ School of Pharmacy, Shanghai Jiao Tong University, Shanghai 200240, China

${ }^{2}$ School of Chemistry and Chemical Engineering, State Key Laboratory of Metal Matrix Composites, Shanghai Jiao Tong University, Shanghai 200240, China

* Corresponding authors (emails: jxcindy@sjtu.edu.cn (Jin X); xyzhu@sjtu.edu.cn (Zhu X)) 
coordination, and $\pi-\pi$ stacking are applied to construct SDPs. Nowadays, SDPs have been widely used in biomedical fields. Although many elegant reviews on SDPs have been published [6-13], to the best of our knowledge, a systematic review on the synthesis and theranostic applications of SDPs has not yet been published.

Herein, we summarize several design and synthesis strategies of SDPs, together with the diagnostic and theranostic-related properties derived from SDPs' structures and highlight the latest theranostic applications. We try to outline these exciting achievements of SDPs for diagnosis, therapy and theranostics, and hope to inspire continuous endeavors in this emerging research field.

\section{DESIGN AND SYNTHESIS}

Viewing from the regularity of branching structure, SDPs systems could be generally divided into supramolecular dendrimers (SDs) [14-18] and supramolecular hyperbranched polymers (SHPs) $[19,20]$. We will briefly summarize the difference in design strategy for SDs and SHPs, highlighting the methodology in synthesis. As increasing demands and interests in supramolecular hybrids, we will also give some discussion on supramolecular hybrid dendritic polymers [21-27] in the end of this section.

\section{Supramolecular dendrimers (SDs)}

In order to achieve regular and highly branched architecture, it usually needs multiple steps and sophisticated design of building blocks to fabricate SDs. Generally, the synthesis methods include template-directed approach and nontemplated approach.

In template-directed approach, branched structures are usually applied as core or subunits, acting as branching template. Zhang and coworkers utilized a three-fold $\beta$ cyclodextrin $\left(\mathrm{CD}_{3}\right)$ as the branching template, linking oligo(ethylene glycol) (OEG) chains via host-guest interaction (Fig. 1). A second-generation supramolecular dendrimer was obtained through dendritic design of the OEG guests [28].

In nontemplated approach, the accuracy of the dendritic structure relies on well-designed, smaller dendritic subunits as building block. The saturability and directionality guarantees the building of polymer in a controllable way. Percec and co-workers have reported various monodendrons by nontemplated approach [29]. Recently, they reported a library of six dendritic monomers containing 12-4EO-PMA and related dendronized polymethacrylates and polyacrylates which were self-assembled with methacrylate or acrylate apex via single-

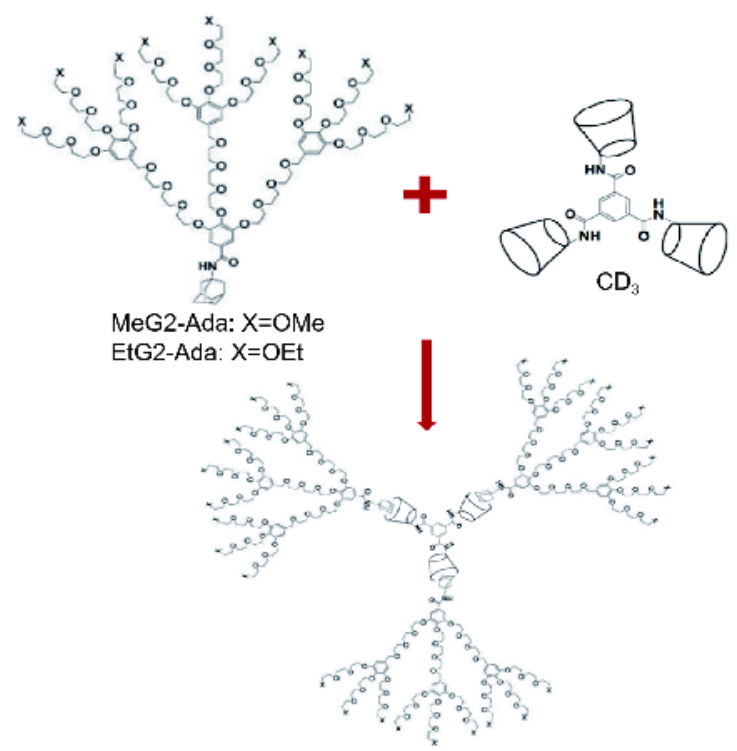

Figure 1 Building blocks and synthesis route for template-directed approach. Reprinted with permission from Ref. [28]. Copyright 2012, Wiley-VCH.

electron transfer living radical polymerization. Zimmerman and coworkers synthesized a dendrimer with complementary donor-donor-acceptor and acceptoracceptor-donor via hydrogen bonding. They got diskshaped particles with very good stability [30].

\section{Supramolecular hyperbranched polymers (SHPs)}

Different from SDs, irregular branched structure is the key factor for SHPs. Feasible synthesis method, such as one-step fabrication, is usually used to fabricate SHPs, resulting in efficient structure-property regulation and various application explorations [31,32]. Single monomer strategy and double-monomer strategy are typical methods for SHPs fabrication.

In single monomer strategy, one heterotropic monomer $\left(\mathrm{AB}_{n}\right.$ type monomer, $\left.n \geq 2\right)$ are used to construct SHPs [33]. Huang and coworkers designed a $\mathrm{AB}_{2}$ monomer containing a bis( $m$-phenylene)-32-crown-10 core and two pendant paraquat arms, driving it to self-recognition to obtain a new SHP [34] (Fig. 2A). In double-monomer strategy, heterocomplementary monomers $\mathrm{A}_{n}$ and $\mathrm{B}_{m}(n$ $\geq 2, m \geq 3$ ) are usually used to obtain SHPs. For example, we reported an $\mathrm{A}_{2} / \mathrm{B}_{3}$ type SHP through host-guest interaction between an azobenzene (Azo) dimer and a $\beta$ $\mathrm{CD}$ trimer (Fig. 2B). Benefiting from the light-responsive building blocks, this SHP is photoreversible between polymerized and depolymerized states under ultraviolet (UV) and visible-light irradiation [35]. 


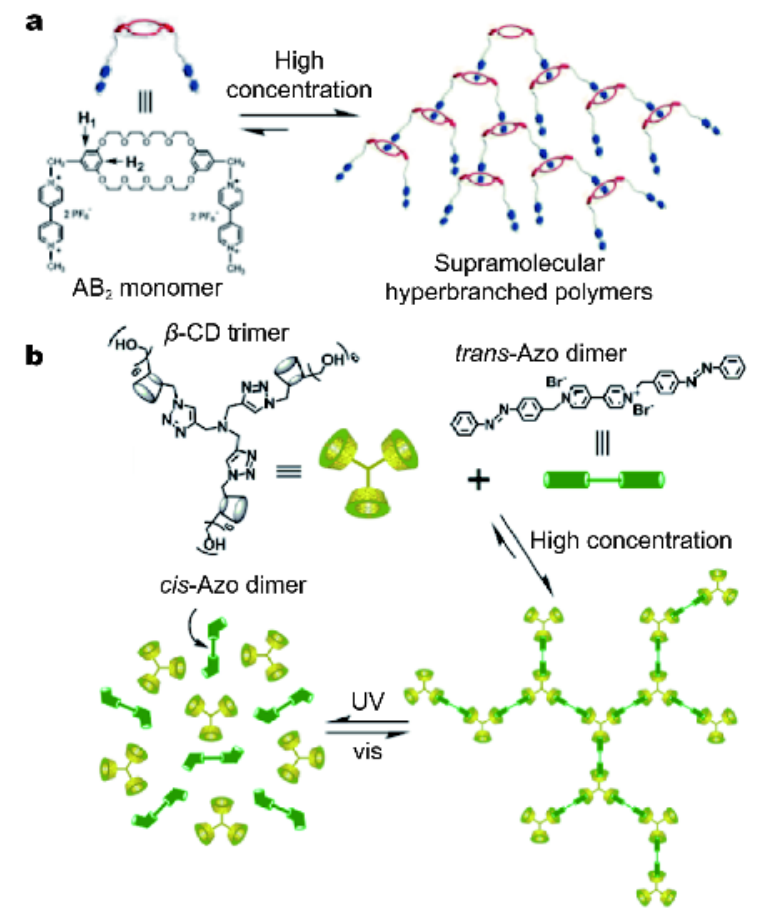

Figure 2 (a) Preparation of $\mathrm{AB}_{2}$-type SHPs through self-recognition. Reprinted with permission from Ref. [34]. Copyright 2004, American Chemical Society. (b) Preparation of photoreversible $\mathrm{A}_{2} / \mathrm{B}_{3}$-type SHPs. Reprinted with permission from Ref. [35]. Copyright 2011, Royal Society of Chemistry.

\section{Supramolecular hybrid dendritic polymers (SHDPs)}

Supramolecular hybrid dendritic polymers (SHDPs) have drawn significant attention in various fields, due to their combination of multiple advantages. Topologically, some SDPs hybridize both advantages of dendritic and linear polymers, forming head-tail like supramolecular hybrids, star-like hybrids, and even more complicated topologic structures. Viewing from component, certain supramolecular systems combine inorganic materials with organic ones, achieving diverse functions. Among various SHDPs, supramolecular hybrid dendrimers own well-defined structure, but the synthesis route always involves multiple procedures. On the other hand, the preparation of supramolecular hybrid hyperbranched polymers is much easier at the expense of structural regularity.

\section{Topological hybrids}

A head-tail like SHDP usually employs a linear part as the tail and a dendritic part as head. Non-covalent interaction is then utilized to link the head and tail together, forming the final hybrids. With the unique topological structures, this type of supramolecular hybrids could be self-assembled into diverse morphologies, including cylinders,

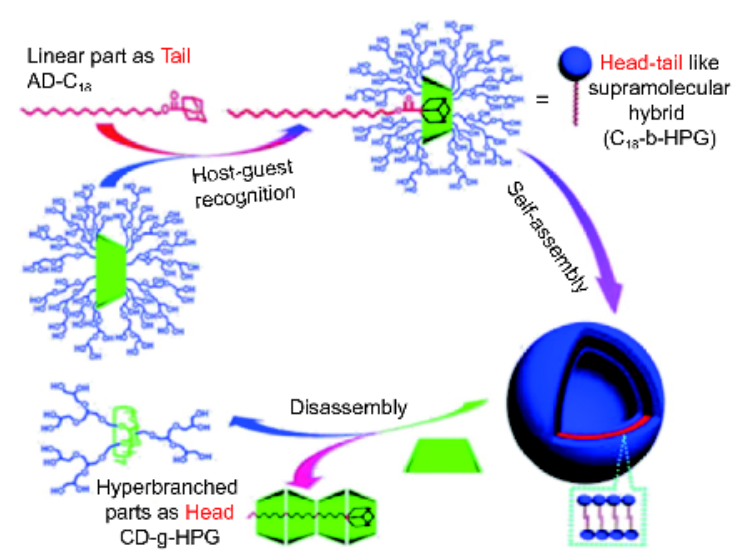

Figure 3 Preparation of supramolecular topological hybrids with headtail shape. Reprinted with permission from Ref. [22]. Copyright 2012, American Chemical Society.

fibers and so on. Zhou, Yan and coworkers [22] designed a hyperbranched polyglycerol grafted from $\beta$-CD (CD-gHPG), and linked a long alkyl chain as tail through hostguest interaction. The resulting supramolecular hybrids self-assembled into unilamellar vesicles in aqueous solution (Fig. 3). Ji and coworkers [36] reported a novel dualresponsive SHDP by utilizing the supramolecular interaction among cucurbit[8]uril (CB[8]) and methyl viologen $(\mathrm{MV})$ and indole. Then, the SHDP self-assembled into micelles and showed dual-responsive abilities when a competitive guest molecule or a reducing agent exists. Kim and Voit [37,38] fabricated a series of compounds containing calix[4]arene core moieties with dendritic polymers on them. These calixarene cores could be recognized by hydrophobic moieties with linear tails, so they are potential to be applied into SHDPs.

Supramolecular star-shape polymers are made of linear polymers as arms as well as a dendritic core with noncovalent linkage, which can also be regarded as a hybrid of polymers with different topology. Three or more arms with identical or distinct polymeric chains radiate from one core to form star-shape polymer. Special properties like various loading capacities and Janus shape could be achieved by novel design of supramolecular star-shape polymers [23,39-43].

\section{Organic-inorganic hybrids}

Besides certain organic molecules, inorganic materials also exhibit strong non-covalent interactions and thus form supramolecular systems $[44,45]$. Metal ions and ligands act with tunable coordination and strong binding strength. They provided accurate coordination number and direction, which makes organic-inorganic supramo- 


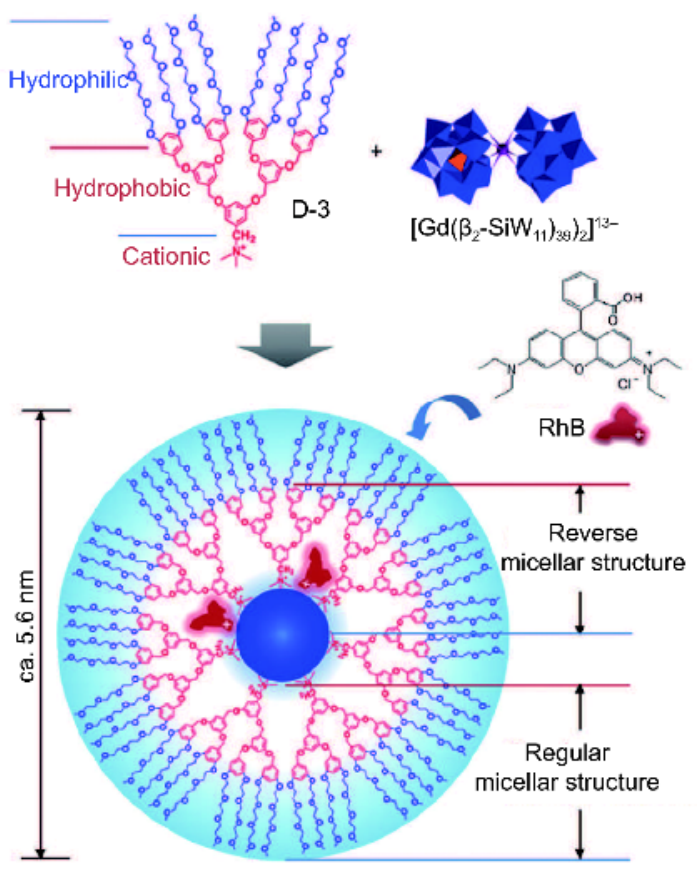

Figure 4 Structure illustration of supramolecular organic-inorganic dendritic hybrid with a poly-oxometalate cluster $\left[\mathrm{Gd}\left(\beta_{2}-\mathrm{SiW}_{11} \mathrm{O}_{39}\right)_{2}\right]^{13-}$ as inorganic core and hyperbranched polymer (D-3) as arms. Reprinted with permission from Ref. [47]. Copyright 2017, Wiley-VCH

lecular dendritic hybrids a popular and useful system in a wide application [14,46]. Li and coworkers [47] developed a novel SDPs with inorganic gadolinium-substituted polyoxometalate as core and organic hyperbranched amphiphilic polymer as arms (Fig. 4). The core and arms could be assembled together through electrostatic interaction. Gu and coworkers [48] reported a self-assembled dual-functionalized low-generation supramolecular hybrid peptide dendrons (PDs) by coordination interactions. The PDs were functionlized with arginine for enhancing internalization and endosomal escape and the core of PDs were modified with lipoic acid (LA) for coordinating potentials.

\section{PROPERTIES AND SELF-ASSEMBLY BEHAVIOR}

\section{Aqueous compatibility and biodegradability}

In nature, most biological processes occur in aqueous system. In this case, good aqueous compatibility is a fundamental factor for SDPs for biological application. However, imaging probes and therapeutic agents are usually of high hydrophobicity. Luckily, various building blocks, such as $\mathrm{CD}$, cucurbit $[n]$ uril and sulfonate calixarene can recognize and include certain hydrophobic guest species $[36,49]$, which provide feasibility to obtain supramolecular system with good aqueous solubility [5053]. Normally, good aqueous compatibility is basic for biocompatibility. The long-term accumulation is another safety concern in biomedical application. Most polymers lose the bio-application chances due to unbreakable conjugation and undegradable property. Supramolecular systems, however, are connected by a dynamic/reversible non-covalent interaction [54-56]. They can be spontaneously degraded or metabolized in physiological environment, exhibiting universally better biodegradability than covalently bonded polymers [57-59].

\section{Stimuli-responsiveness}

During biological use, especially in diagnosis and therapy, fast and smart stimuli-responsiveness is highly favored, because the environmental factors in living system could be altered in very limited scope. SDPs, which can respond to internal stimuli such as $\mathrm{pH}[50,51]$, redox [14], voltage [50] and enzyme [53], are usually exhibiting good release behavior or high contrast signals when using as delivery vehicles for drugs or imaging probes. At the same time, the ones responsive to external stimuli such as temperature $[30,31,56]$, light $[12,35,55]$, ultrasonic signal and magnetic field, can be fabricated into smart in vitro diagnosis or therapeutic devices [60-68].

\section{Self-assembly behavior}

Similar to traditional polymer systems, the main driving force for SDPs to self-assembly is hydrophobic interaction [65]. As a dynamic switching system, however, a typical SDPs self-assembly process usually involves two stages, firstly forming primary structure through supramolecular interaction then obtaining secondary structure through self-assembling [66]. Both of the above stages rely on non-covalent interactions. In order to achieve clear and stable primary structure as well as secondary structure, stronger interactions are usually employed in supramolecular stage. Through sophisticated design of the supramolecular system, diverse morphologies can be achieved including spherical micelles, cylindrical micelles, fibers, nano-rings and tubular vesicles [15-18,22,36, $37,67]$.

\section{BIOMEDICAL APPLICATIONS}

Combining both advantages of dendritic polymers and supramolecular polymers, SDPs have shown great potential in biomedical application. With a three-dimensional globular topological structures, SDP own plenty of inner cavities and terminal groups, and therefore their 
loading capacity and surface functions can be easily regulated. Favored functions in biomedical application, such as imaging probe loading, drug loading, targeted surface and stimuli-response, can be designed and integrated into one SDP [23]. Benefitting from the dynamic/tunable nature of non-covalent interactions, SDPs can undergo reversible switching of morphology, structures and functions. In this section, we will introduce several eye-catching studies to indicate new trends of biomedical application of SDPs in bio-imaging and diagnostic, therapeutic and theranostic applications [69$71]$.

\section{Bio-imaging and diagnosis}

Benefitting from the topological structures, SDPs are able to load various imaging probes, which provide safe and favored imaging environment in vitro and in vivo. Up to now, plenty of types of imaging probes, including fluorescent probes [42], ultrasound [72] and magnetic resonance imaging (MRI) contrast agents [73-75], and NIR imaging probes [74], have been successfully integrated in SDPs for bio-imaging and diagnosis.

$\mathrm{Wu}$ and coworkers reported a novel SDP for in vivo MRI imaging [75]. An inorganic metal-oxide nanocluster polyoxometalate (POM) was utilized as both Gd ligand and template. Tri(ethylene glycol) monomethyl ether (TEG) terminal groups in two generation was used to encapsulate Gd-POM through electrostatic interaction, which provides in vivo stability as well as good water permeation for higher relaxivity of $\mathrm{Gd}$, resulting in enhanced in vivo imaging capacity (Fig. 5).

\section{Therapeutic application}

Controllable release of loading therapeutics is an ultimate goal for drug delivery system, and therefore an integrated delivery system is favored. Covalent conjugation based systems, however, naturally possess low degradation behavior, which causes the poor permeation into biological barriers and low release rate of therapeutics [77-79]. Benefitting from the dynamic/reversible feature, SDPs have been regarded as a promising platform for therapeutics delivery.

A large number of SDPs have been applied to load pharmaceutical agents, including chemo-drugs $[80,81]$ and photosensitizers [82], resulting in optimized delivery and releasing behaviors like prolonged circulation time, accumulation in tumor sites, controlled drug release and reduced side effects. A significant reason for above phenomenon is that SDPs are built via non-covalent interaction and able to rapidly respond to environmental

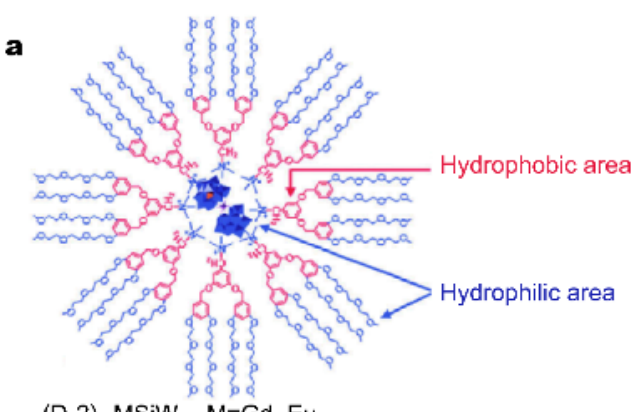

$(\mathrm{D}-2)_{13} \mathrm{MSiW}_{11}, \mathrm{M}=\mathrm{Gd}, \mathrm{Eu}$

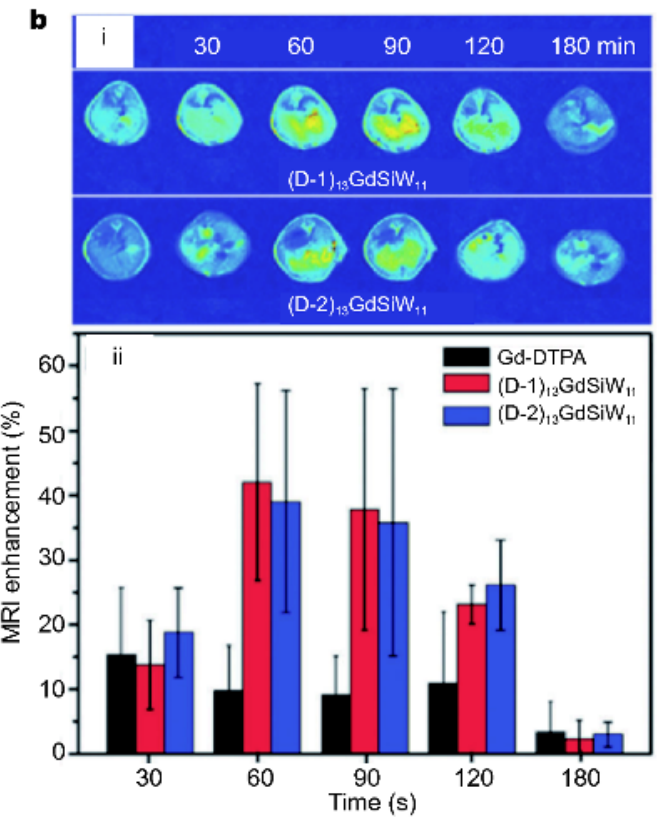

Figure 5 (a) Structure illustration of MRI contrast agent Gd-loaded SDPs with hydrophobic core and hydrophilic arms. (b) T1-weighted images (i) and columnar plots of mean MRI enhancement efficiency (ii) of at liver after post intravenous injection of SDPs. Reprinted with permission from Ref. [75]. Copyright 2017, Royal Society of Chemistry.

\section{changes.}

Other than drug delivery, gene delivery is equally important in treating diseases, and non-viral delivery systems based on cationic materials have gained much attention [83,84]. Cationic materials based on covalent interaction always suffer from several disadvantages, including tedious synthesis and lack of responsiveness [8587]. SDPs offer new opportunities for novel gene delivery vehicles, with dynamic cationic structure and tunable performance. Up to now, different kinds of genetic therapeutics, including DNA [88], siRNA [89] and interfering RNA [90] have been successfully compacted with SDPs, showing comparable gene transfection efficiency with covalent systems.

Our group has developed a facile approach for charge- 

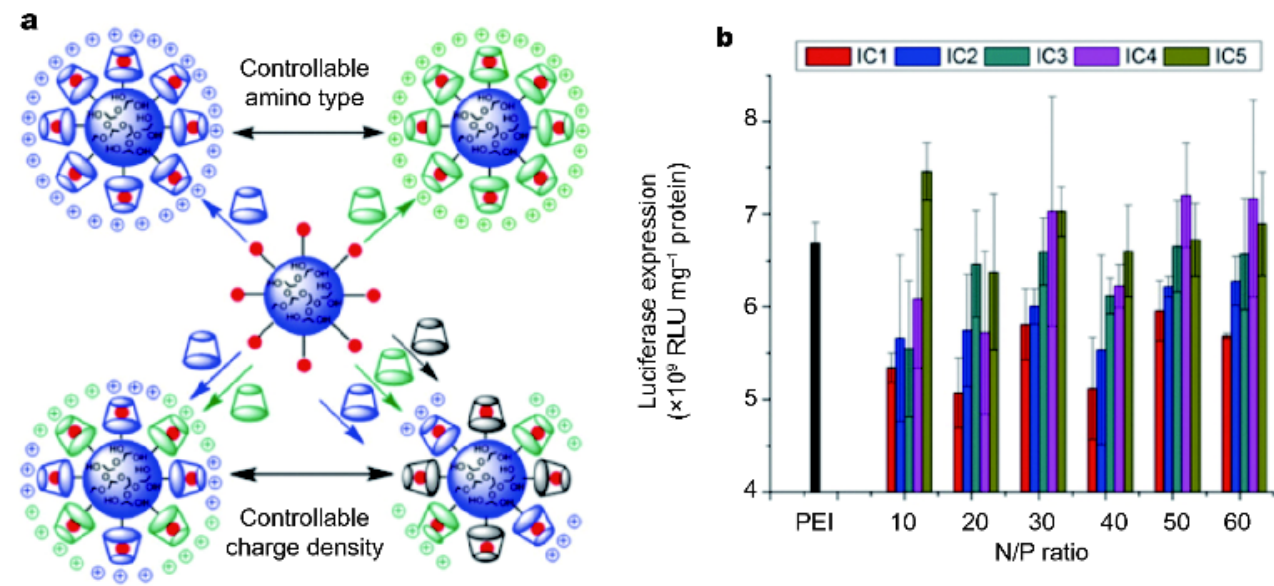

Figure 6 (a) Structure illustration of cationic SDPs with controllable amine type and charge density. (b) Luciferase expression of cationic SDPs in COS-7 cell line. Reprinted with permission from Ref. [43]. Copyright 2011, Royal Society of Chemistry.

tunable cationic SDPs for gene delivery. This system is composed of adamantane-modified hyperbranched polyglycerol (HPG), primary-amine- and tertiary-aminemodified $\beta$-CD derivatives (Fig. 6). By regulation of feed ratio of two cationic $\beta$-CD building blocks, controllable amino types and controllable charge density could be achieved, which further influenced the DNA condensation capability and buffer capacity, together with gene transfection efficiency [43].

\section{Theranostic application}

Theranostic system combining diagnosis with therapy together, is a favored approach in clinical application. These two factors can coordinate with each other, providing integrated information on disease location, drug release profile and treatment feedbacks, all of which are suggestive for further treatment.

SDP-based theranostic system is an elegant combination of supramolecular chemistry, topo-chemical science, imaging science and pharmaceutical science. SDPs are usually constructed from multiple non-covalent interactions, providing diverse and suitable physical/chemical environment for imaging probes and therapeutics. A number of pilot studies has proved its high efficiency and practicability $[49,78,79,83]$. Gu and coworkers [76] reported a platinum-based supramolecular dendritic system for efficient delivery of chemo-drug and near-infrared (NIR) agents. Supramolecular dendritic systems were stabilized by disulfide bonds and endowed with NIR fluorescence probes. Then PEGylated platinum derivatives were coordinated onto the abundant peripheral groups of supramolecular dendritic templates to generate
$\mathrm{pH} /$ redox dual-responsive theranostic supramolecular PEGylated dendritic systems (Fig. 7).

\section{CONCLUSIONS}

In this review, we have summarized the current progress in the preparation and theranostic applications of SDPs. From structural perspective, both non-covalent interactions and three-dimensional topological structures play an important role in the physicochemical properties of SDPs. Owing to the introduction of non-covalent interaction into dendritic polymeric backbone, the SDPs not only display dynamic regulation of specific structures and functions when exposed to external stimuli, but also keep the advantages of dendritic polymers. Correspondingly, SDPs exhibit the abilities of facile synthesis, good biocompatibility, inherent biodegradability, smart responsiveness, simple metabolism, and scaled preparation, which are very attractive for biomedical science. Therefore, SDPs have become an excellent platform for the diagnostic and theranostic applications.

Although great progress has been made in the field of SDPs for diagnostic and theranostic applications, this research field is still far away from sufficient and continuous efforts are particularly required. For example, the construction methods of SDPs are quite limited and current SDPs are simple and monotone. The excretory and metabolic pathways of SDPs in cells and body are not reported and the molecular interactions between SDPs and cells is unclear. More importantly, all SDPs investigations focus on the cell evaluation and mice experiments. To meet the requirements of clinical trial, the design of biocompatible and biodegradable SDPs with 

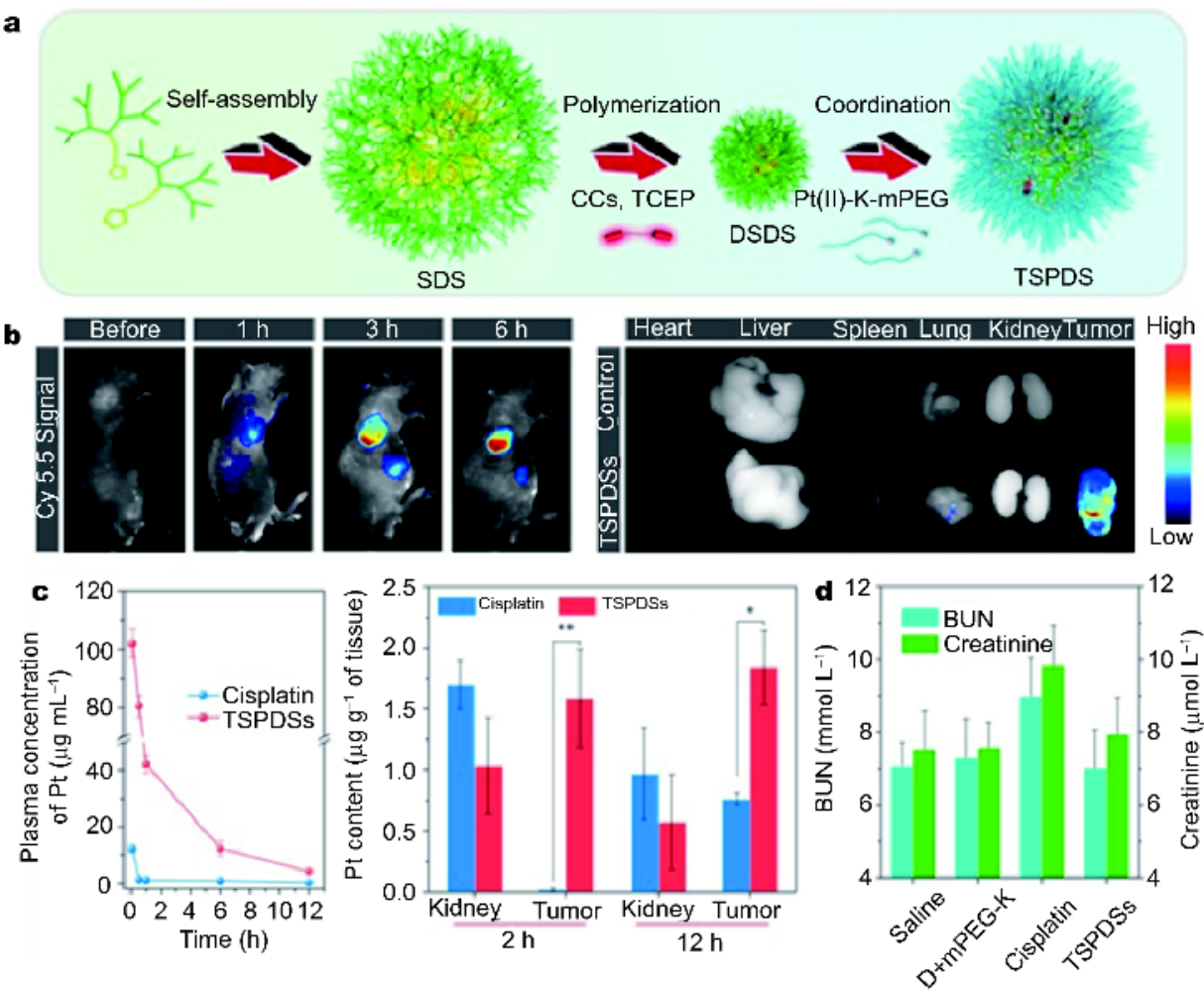

Figure 7 (a) Schematic illustrations for molecular and supramolecular engineering of theranostic supramolecular PEGylated dendritic systems. (b) In vivo and ex vivo NIR imaging of nude mice bearing A549 tumor xenografts and their organs before and after 24 hours intravenous injection of saline and TSPDSs. (c) Pharmacokinetic profiles of TSPDSs and cisplatin and distribution of platinum in kidney and tumor after intravenous injection, respectively. (d) Blood urea nitrogen (BUN) and creatinine level with different administrations after a treatment course of 21 days. Reprinted with permission from Ref. [76]. Copyright 2016, Ivyspring International Publisher.

precise structures have become very urgent and important. Frankly speaking, the process of paving SDPs into diagnostic and theranostic clinical applications will be long and full of difficulties. Nevertheless, we believe that with the rapid development in this research field, the present problems will be resolved in the near future.

Received 10 February 2018; accepted 17 March 2018; published online 16 April 2018

1 Voit BI. Dendritic polymers: From aesthetic macromolecules to commercially interesting materials. Acta Polym, 1995, 46: 87-99

2 Ma Y, Mou Q, Wang D, et al. Dendritic polymers for theranostics. Theranostics, 2016, 6: 930-947

3 Gao C, Yan D. Hyperbranched polymers: from synthesis to applications. Prog Polymer Sci, 2004, 29: 183-275

4 Shcharbin D, Janaszewska A, Klajnert-Maculewicz B, et al. How to study dendrimers and dendriplexes III. Biodistribution, pharmacokinetics and toxicity in vivo. J Control Release, 2014, 181: 40-52

5 Mishra V, Gupta U, Jain NK. Surface-engineered dendrimers: a solution for toxicity issues. J BioMater Sci Polymer Ed, 2009, 20: 141-166

6 Dong R, Zhou Y, Zhu X. Supramolecular dendritic polymers: from synthesis to applications. Acc Chem Res, 2014, 47: 2006-2016

7 Dong R, Zhou Y, Huang X, et al. Functional supramolecular polymers for biomedical applications. Adv Mater, 2015, 27: 498526

8 Zhou J, Yu G, Huang F. Supramolecular chemotherapy based on host-guest molecular recognition: a novel strategy in the battle against cancer with a bright future. Chem Soc Rev, 2017, 46: 70217053

9 Yan X, Wang F, Zheng B, et al. Stimuli-responsive supramolecular polymeric materials. Chem Soc Rev, 2012, 41: 6042-6065

10 Awani Kumar Rai RT, Priyanka Maurya, Pooja Yadav. Dendrimers: A potential carrier for targeted drug delivery system. Pharm Biol Eval, 2016, 3 : 275-287

11 Yu G, Jie K, Huang F. Supramolecular amphiphiles based on hostguest molecular recognition motifs. Chem Rev, 2015, 115: 72407303

12 Liu Y, Yu C, Jin H, et al. A supramolecular janus hyperbranched polymer and its photoresponsive self-assembly of vesicles with narrow size distribution. J Am Chem Soc, 2013, 135: 4765-4770

13 Tian W, Li X, Wang J. Supramolecular hyperbranched polymers. Chem Commun, 2017, 53: 2531-2542

14 Vögtle F, Plevoets M, Nieger M, et al. Dendrimers with a photoactive and redox-active $\left[\mathrm{Ru}(\mathrm{bpy})_{3}\right]^{2+}$-type core: photophysical properties, electrochemical behavior, and excited-state electron- 
transfer reactions. J Am Chem Soc, 1999, 121: 6290-6298

15 Franz A, Bauer W, Hirsch A. Complete self-assembly of discrete supramolecular dendrimers. Angew Chem Int Ed, 2005, 44: 15641567

16 Xie D, Jiang M, Zhang G, et al. Hydrogen-bonded dendronized polymers and their self-assembly in solution. Chem Eur J, 2007, 13: 3346-3353

17 Leung KCF, Mendes PM, Magonov SN, et al. Supramolecular selfassembly of dendronized polymers: reversible control of the polymer architectures through acid-base reactions. J Am Chem Soc, 2006, 128: 10707-10715

18 Kim Y, Mayer MF, Zimmerman SC. A new route to organic nanotubes from porphyrin dendrimers. Angew Chem Int Ed, 2003, 42: $1121-1126$

19 Liu Q, Zhang H, Yin S, et al. Hierarchical self-assembling of dendritic-linear diblock complex based on hydrogen bonding. Polymer, 2007, 48: 3759-3770

20 Alvarez-Parrilla E, Ramos Cabrer P, Al-Soufi W, et al. Dendritic growth of a supramolecular complex. Angew Chem Int Ed, 2000, 39: 2856-2858

21 Zou J, Guan B, Liao X, et al. Dual reversible self-assembly of PNIPAM-based amphiphiles formed by inclusion complexation. Macromolecules, 2009, 42: 7465-7473

22 Tao W, Liu Y, Jiang B, et al. A linear-hyperbranched supramolecular amphiphile and its self-assembly into vesicles with great ductility. J Am Chem Soc, 2012, 134: 762-764

23 Andrén OCJ, Zhang Y, Lundberg P, et al. Therapeutic nanocarriers via cholesterol directed self-assembly of well-defined linear-dendritic polymeric amphiphiles. Chem Mater, 2017, 29: 3891-3898

24 Gillies ER, Fréchet JMJ. Synthesis and self-assembly of supramolecular dendritic "bow-ties": effect of peripheral functionality on association constants. J Org Chem, 2004, 69: 46-53

25 Demattei CR, Huang B, Tomalia DA. Designed dendrimer syntheses by self-assembly of single-site, ssDNA functionalized dendrons. Nano Lett, 2004, 4: 771-777

26 Precup-Blaga FS, Garcia-Martinez JC, Schenning APHJ, et al. Highly emissive supramolecular oligo( $p$-phenylene vinylene) dendrimers. J Am Chem Soc, 2003, 125: 12953-12960

27 Chechik V, Zhao M, Crooks RM. Self-assembled inverted micelles prepared from a dendrimer template: phase transfer of encapsulated guests. J Am Chem Soc, 1999, 121: 4910-4911

28 Yan J, Zhang X, Zhang X, et al. Thermoresponsive supramolecular dendrimers via host-guest interactions. Macromol Chem Phys, 2012, 213: 2003-2010

29 Andreopoulou KA, Peterca M, Wilson DA, et al. Demonstrating the $8_{1}$-helicity and nanomechanical function of self-organizable dendronized polymethacrylates and polyacrylates. Macromolecules, 2017, 50: 5271-5284

30 Ma Y, Kolotuchin SV, Zimmerman SC. Supramolecular polymer chemistry: self-assembling dendrimers using the DDA.AAD (GClike) hydrogen bonding motif. J Am Chem Soc, 2002, 124: 1375713769

31 Yan J, Li W, Liu K, et al. Thermoresponsive supramolecular dendronized polymers. Chem Asian J, 2011, 6: 3260-3269

32 Yin JF, Hu Y, Wang DG, et al. Cucurbit[8] uril-based water-soluble supramolecular dendronized polymer: evidence from single polymer chain morphology and force spectroscopy. ACS Macro Lett, 2017, 6: 139-143

33 Wang X, Yang Y, Fan L, et al. POSS-embedded supramolecular hyperbranched polymers constructed from a $1 \rightarrow 7$ branching monomer with controllable morphology transitions. Sci China Chem, 2018, 61: 311-318

34 Huang F, Gibson HW. Formation of a supramolecular hyperbranched polymer from self-organization of an $\mathrm{AB}_{2}$ monomer containing a crown ether and two paraquat moieties. J Am Chem Soc, 2004, 126: 14738-14739

35 Dong R, Liu Y, Zhou Y, et al. Photo-reversible supramolecular hyperbranched polymer based on host-guest interactions. Polym Chem, 2011, 2: 2771

36 Chen CJ, Li DD, Wang HB, et al. Fabrication of dual-responsive micelles based on the supramolecular interaction of cucurbit[8] uril. Polym Chem, 2013, 4: 242-245

37 Roy R, Kim JM. Amphiphilicp-tert-butylcalix[4] arene scaffolds containing exposed carbohydrate dendrons. Angew Chem Int Ed, 1999, 38: 369-372

38 Appelhans D, Smet M, Khimich G, et al. Lysine dendrimers based on thiacalix[4]arene core moieties as molecular scaffolds for supramolecular host systems. New J Chem, 2005, 29: 1386-1389

39 Choi Y, Mecke A, Orr BG, et al. DNA-directed synthesis of generation 7 and 5 PAMAM dendrimer nanoclusters. Nano Lett, 2004, 4: 391-397

40 Kaewtong C, Jiang G, Felipe MJ, et al. Self-assembly and electrochemical oxidation of polyamidoamine-carbazole dendron surfmer complexes: nanoring formation. ACS Nano, 2008, 2: 15331542

41 Wang D, Chen H, Su Y, et al. Supramolecular amphiphilic multiarm hyperbranched copolymer: synthesis, self-assembly and drug delivery applications. Polym Chem, 2013, 4: 85-94

42 Vergara J, Gimeno N, Cano M, et al. Mesomorphism from bentcore based ionic dendritic macromolecules. Chem Mater, 2011, 23: 4931-4940

43 Dong R, Zhou L, Wu J, et al. A supramolecular approach to the preparation of charge-tunable dendritic polycations for efficient gene delivery. Chem Commun, 2011, 47: 5473-5475

$44 \mathrm{Xu} \mathrm{X,} \mathrm{Lu} \mathrm{Y,} \mathrm{Yang} \mathrm{Y,} \mathrm{et} \mathrm{al.} \mathrm{Tuning} \mathrm{the} \mathrm{growth} \mathrm{of} \mathrm{metal-organic}$ framework nanocrystals by using polyoxometalates as coordination modulators. Sci China Mater, 2015, 58: 370-377

45 Gao ZC, Wei CP, Han YF, et al. Near-infrared-emissive self-assembled polymers via the implementation of molecular tweezer/ guest complexation on a supramolecular coordination complex platform. Chin J Polym Sci, 2018, 36: 399-405

46 Daniel MC, Ruiz J, Astruc D. Supramolecular H-bonded assemblies of redox-active metallodendrimers and positive and unusual dendritic effects on the recognition of $\mathrm{H}_{2} \mathrm{PO}_{4}^{-}$. J Am Chem Soc, 2003, 125: 1150-1151

47 Zhang S, Zheng Y, Yin S, et al. A dendritic supramolecular complex as uniform hybrid micelle with dual structure for bimodal in vivo imaging. Chem Eur J, 2017, 23: 2802-2810

$48 \mathrm{Xu} \mathrm{X}$, Jian Y, Li Y, et al. Bio-inspired supramolecular hybrid dendrimers self-assembled from low-generation peptide dendrons for highly efficient gene delivery and biological tracking. ACS Nano, 2014, 8: 9255-9264

49 Ge Z, Liu H, Zhang Y, et al. Supramolecular thermoresponsive hyperbranched polymers constructed from poly $(N$-isopropylacrylamide) containing one adamantyl and two $\beta$-cyclodextrin terminal moieties. Macromol Rapid Commun, 2011, 32: 68-73

50 Fernández G, Pérez EM, Sánchez L, et al. An electroactive dynamically polydisperse supramolecular dendrimer. J Am Chem Soc, 2008, 130: 2410-2411 
51 Yu B, Wang B, Guo S, et al. pH-controlled reversible formation of a supramolecular hyperbranched polymer showing fluorescence switching. Chem Eur J, 2013, 19: 4922-4930

52 Liu Y, Huang Z, Liu K, et al. Porphyrin-containing hyperbranched supramolecular polymers: enhancing ${ }^{1} \mathrm{O}_{2}$-generation efficiency by supramolecular polymerization. Polym Chem, 2014, 5: 53-56

53 Yu S, Zhang W, Zhu J, et al. Construction of a hyperbranched supramolecular polymer as a bifunctional antioxidative enzyme model. Macromol Biosci, 2011, 11: 821-827

54 Jeon YJ, Bharadwaj PK, Choi SW, et al. Supramolecular amphiphiles: spontaneous formation of vesicles triggered by formation of a charge-transfer complex in a host. Angew Chem Int Ed, 2002, 41: $4474-4476$

55 Sun R, Xue C, Ma X, et al. Light-driven linear helical supramolecular polymer formed by molecular-recognition-directed self-assembly of bis( $p$-sulfonatocalix[4]arene) and pseudorotaxane. J Am Chem Soc, 2013, 135: 5990-5993

56 Wang C, Wang Z, Zhang X. Superamphiphiles as building blocks for supramolecular engineering: towards functional materials and surfaces. Small, 2011, 7: 1379-1383

57 Dong S, Zheng B, Wang F, et al. Supramolecular polymers constructed from macrocycle-based host-guest molecular recognition motifs. Acc Chem Res, 2014, 47: 1982-1994

58 Ma X, Tian H. Stimuli-responsive supramolecular polymers in aqueous solution. Acc Chem Res, 2014, 47: 1971-1981

59 Ooya T, Ito A, Yui N. Preparation of a-cyclodextrin-terminated polyrotaxane consisting of $\beta$-cyclodextrins and pluronic as a building block of a biodegradable network. Macromol Biosci, 2005, 5: $379-383$

60 Nakahata M, Takashima Y, Yamaguchi H, et al. Redox-responsive self-healing materials formed from host-guest polymers. Nat Commun, 2011, 2: 511

61 Williams RJ, Smith AM, Collins R, et al. Enzyme-assisted selfassembly under thermodynamic control. Nat Nanotechnol, 2009, 4: $19-24$

62 Xing Y, Wang C, Han P, et al. Acetylcholinesterase responsive polymeric supra-amphiphiles for controlled self-assembly and disassembly. Langmuir, 2012, 28: 6032-6036

63 Seo JH, Kakinoki S, Inoue Y, et al. Inducing rapid cellular response on RGD-binding threaded macromolecular surfaces. J Am Chem Soc, 2013, 135: 5513-5516

64 Jiang W, Kim BYS, Rutka JT, et al. Nanoparticle-mediated cellular response is size-dependent. Nat Nanotechnol, 2008, 3: 145-150

65 Hyun H, Yui N. Ligand accessibility to receptor binding sites enhanced by movable polyrotaxanes. Macromol Biosci, 2011, 11: 765-771

66 Thota BNS, Urner LH, Haag R. Supramolecular architectures of dendritic amphiphiles in water. Chem Rev, 2015, 116: 2079-2102

67 Kiessling LL, Gestwicki JE, Strong LE. Synthetic multivalent ligands as probes of signal transduction. Angew Chem Int Ed, 2006, 45: $2348-2368$

68 Wang D, Tong G, Dong R, et al. Self-assembly of supramolecularly engineered polymers and their biomedical applications. Chem Commun, 2014, 50: 11994-12017

69 Kim HM, Cho BR. Two-photon probes for intracellular free metal ions, acidic vesicles, and lipid rafts in live tissues. Acc Chem Res, 2009, 42: 863-872

70 Morawski AM, Lanza GA, Wickline SA. Targeted contrast agents for magnetic resonance imaging and ultrasound. Curr Opin Biotech, 2005, 16: 89-92
71 Janib SM, Moses AS, MacKay JA. Imaging and drug delivery using theranostic nanoparticles. Adv Drug Deliver Rev, 2010, 62: 10521063

72 Li N, Jin Y, Xue L, et al. ${ }^{188}$ Re-labeled hyperbranched polysulfonamine as a robust tool for targeted cancer diagnosis and radioimmunotherapy. Chin J Polym Sci, 2013, 31: 530-540

73 Chen KJ, Wolahan SM, Wang $\mathrm{H}$, et al. A small MRI contrast agent library of gadolinium(III)-encapsulated supramolecular nanoparticles for improved relaxivity and sensitivity. Biomaterials, 2011, 32: $2160-2165$

74 Saatchi K, Gelder N, Gershkovich P, et al. Long-circulating nontoxic blood pool imaging agent based on hyperbranched polyglycerols. Int J Pharm, 2012, 422: 418-427

75 Zhang S, Zheng Y, Fu DY, et al. Biocompatible supramolecular dendrimers bearing a gadolinium-substituted polyanionic core for MRI contrast agents. J Mater Chem B, 2017, 5: 4035-4043

76 Li Y, Li Y, Zhang X, et al. Supramolecular PEGylated dendritic systems as $\mathrm{pH} /$ redox dual-responsive theranostic nanoplatforms for platinum drug delivery and NIR imaging. Theranostics, 2016, 6: 1293-1305

77 Davis ME, Brewster ME. Cyclodextrin-based pharmaceutics: past, present and future. Nat Rev Drug Discov, 2004, 3: 1023-1035

78 Li J, Loh XJ. Cyclodextrin-based supramolecular architectures: Syntheses, structures, and applications for drug and gene delivery. Adv Drug Deliver Rev, 2008, 60: 1000-1017

79 Zhang J, Ma PX. Cyclodextrin-based supramolecular systems for drug delivery: Recent progress and future perspective. Adv Drug Deliver Rev, 2013, 65: 1215-1233

80 Huang D, Wang Y, Yang F, et al. Charge-reversible and pH-responsive biodegradable micelles and vesicles from linear-dendritic supramolecular amphiphiles for anticancer drug delivery. Polym Chem, 2017, 8: 6675-6687

81 Zhang Y, Xiao C, Li M, et al. Co-delivery of 10-hydroxycamptothecin with doxorubicin conjugated prodrugs for enhanced anticancer efficacy. Macromol Biosci, 2013, 13: 584-594

82 Liu F, Zhang Y, Pan X, et al. Doxorubicin-loaded redox-responsive amphiphilic dendritic porphyrin conjugates for chemotherapy and photodynamic therapy. RSC Adv, 2016, 6: 57552-57562

83 Mintzer MA, Simanek EE. Nonviral vectors for gene delivery. Chem Rev, 2009, 109: 259-302

84 Schaffert D, Wagner E. Gene therapy progress and prospects: synthetic polymer-based systems. Gene Ther, 2008, 15: 1131-1138

85 Chen Y, Zhou L, Pang Y, et al. Photoluminescent hyperbranched poly(amido amine) containing $\beta$-cyclodextrin as a nonviral gene delivery vector. Bioconjugate Chem, 2011, 22: 1162-1170

86 Shi Y, Du J, Zhou L, et al. Size-controlled preparation of magnetic iron oxidenanocrystals within hyperbranched polymers and their magnetofection in vitro. J Mater Chem, 2012, 22: 355-360

87 Wang R, Zhou L, Zhou Y, et al. Synthesis and gene delivery of poly (amido amine)s with different branched architecture. Biomacromolecules, 2010, 11: 489-495

88 Wang $\mathrm{H}$, Liu K, Chen KJ, et al. A rapid pathway toward a superb gene delivery system: programming structural and functional diversity into a supramolecular nanoparticle library. ACS Nano, 2010, 4: 6235-6243

89 Chen $\mathrm{H}$, Jia H, Tham HP, et al. Theranostic prodrug vesicles for imaging guided codelivery of camptothecin and siRNA in synergetic cancer therapy. ACS Appl Mater Interfaces, 2017, 9: 2353623543

90 Chen C, Posocco P, Liu X, et al. Mastering dendrimer self-as- 
sembly for efficient sirna delivery: from conceptual design to in vivo efficient gene silencing. Small, 2016, 12: 3667-3676

Acknowledgements This work was financially supported by the National Natural Science Foundation of China (51690151, 51503122, 21374062 and 51473093) and National Basic Research Program (2015CB931801).
Author contributions $\mathrm{Zhu} \mathrm{X}$ and Jin $\mathrm{X}$ provided the whole concept. Zhu $\mathrm{X}$ wrote the abstract, introduction and conclusion sections. Jin $\mathrm{X}$ wrote the synthesis, properties and self-assembly and biomedical application sections. Wu Y summarized the references. Xu G revised the manuscript. All authors contributed to the general discussion.

Conflict of interest The authors declare no conflict of interest.

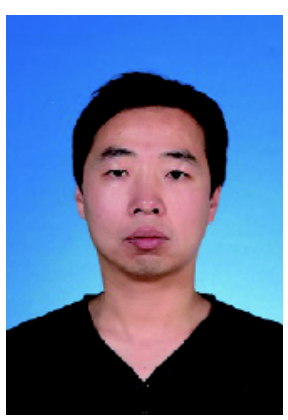

Yan Wu completed his BSc degree at Baoji University of Arts and Sciences in 2010 and then earned his MSc degree at Shantou University in 2013. Now, he is pursuing his PhD degree at the School of Pharmacy, Shanghai Jiao Tong University under the supervision of Prof. Xinyuan Zhu and Prof. Dawei Li. His current interests focus on the preparation of functional polymers as well as their applications in biomedical fields.
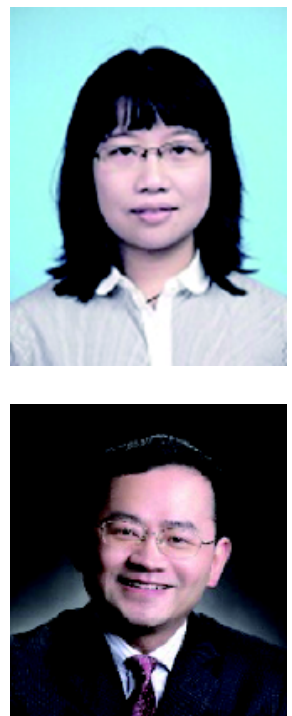

Xin Jin completed her BSc degree in Chemistry at Wuhan University in 2007. She obtained PhD degree in Polymer Physics \& Chemistry at Institute of Chemistry, Chinese Academy of Sciences in 2013. She continued her academic research as a post-doctoral fellow in Shanghai Jiao Tong University. Currently, she is an assistant professor in Shanghai Jiao Tong University and her research interests include modification and application of hyperbranched polymers, natural polymers and polyelectrolytes.

\section{超分子枝状聚合物用于疾病诊断与治疗}

吴演 ${ }^{1}$ 许冠哲 ${ }^{2}$, 金垔 $^{2^{*}}$, 朱新远 $^{2^{*}}$

摘要 超分子枝状聚合物(SDP)为疾病的诊断与治疗提供了新的方法. 这种聚合物具有非共价链接的大分子与枝状结构以及三维管状结 构. 这种结构形成了动态可逆的理化特性(如高溶解性, 粘度低以及富含大量的功能团端基). 可逆的非共价相互作用使得SDP易于制备, 具有智能响应以及良好的代谢性. 这些特性决定了SDP在疾病诊断与治疗中起到关键性作用. 本综述简要总结了具有特定结构、性质和 功能的SPD分子的合成与设计. SPD在诊断治疗中的应用促进了化学、材料科学以及生物医学的交叉研究. 